Saudi Journal of Oral and Dental Research

Abbreviated Key Title: Saudi J Oral Dent Res

ISSN 2518-1300 (Print) |ISSN 2518-1297 (Online)

Scholars Middle East Publishers, Dubai, United Arab Emirates

Journal homepage: https://saudijournals.com/sjodr

\title{
Clinical and Radiological Approaches of Calcifying Odontogenic Cyst of Maxilla - A Case Report
}

\author{
Dr. S. Jayachandran MDS, PhD, MAMS, FDSRCPS (Glasg) $)^{1}$, Dr. A.V. Annapoorni ${ }^{2 *}$ \\ ${ }^{1}$ Department of Oral Medicine and Radiology Tamil Nadu Government Dental College and Hospital, Affiliated to the Tamil Nadu Dr.M.G.R. Medical \\ University Chennai, India \\ ${ }^{2}$ Post Graduate student Department of Oral Medicine and Radiology Tamil Nadu Government Dental College and Hospital Affiliated to The Tamil \\ Nadu Dr.M.G.R. Medical University Chennai , India
}

DOI: $10.36348 /$ sjodr.2020.v05i06.009

| Received: 20.06.2020 | Accepted: 27.06.2020 | Published: 30.06.2020

*Corresponding author: Dr A.V. Annapoorni

\section{Abstract}

Calcifying Odontogenic cyst (COC) or Gorlin cyst is an uncommon benign cystic lesion which develops from the odontogenic epithelium. According to WHO it is classified under developmental Odontogenic cysts. They occupy a spectrum ranging from a cyst to an odontogenic tumor. It most commonly involves the anterior region of the jaws. The characteristic feature of COC is the presence of Ghost cells within the epithelium that has a propensity to calcify. In this paper we illustrate a case of COC in a 14-year-old girl, and the investigations done to arrive at the final diagnosis.

Keywords: Gorlin Cyst, Odontogenic Cysts, calcifying, Maxillary antrum, diagnosis, jaw cyst.

Copyright @ 2020: This is an open-access article distributed under the terms of the Creative Commons Attribution license which permits unrestricted use, distribution, and reproduction in any medium for non-commercial use (NonCommercial, or CC-BY-NC) provided the original author and source are credited.

\section{INTRODUCTION}

COC was described by Gorlin et al in 1962[1]. It is an unusual lesion which contributes to nearly $1 \%$ of the jaw cysts. It most commonly involves the incisor canine areas and rarely involves posterior maxilla [2].The World Health organisation in 2005 described $\mathrm{COC}$ as a benign cystic tumor which arises from the odontogenic epithelium with or without hard tissue formation, but later in 2017 they reclassified COC under developmental odontogenic cysts[3].The characteristic feature of COC is the presence of Ghost cells within the epithelium that tend to calcify[ 4].these lesions occur most commonly in the second decade of life with equal susceptibility to both the genders. It can
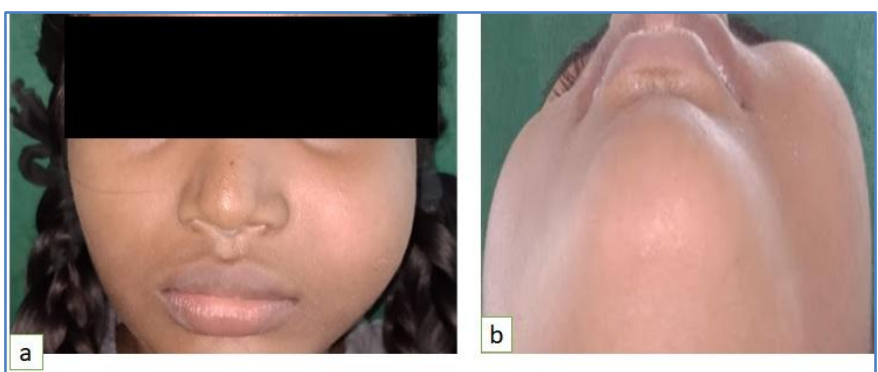

Figure 1 be either intraosseous (central) or extraosseous (peripheral) [5].

\section{CASE REPORT}

A 14-year-old female patient reported to the department of Oral Medicine and Radiology with a complaint of pain and swelling in the left upper jaw for past 2 months. On eliciting the history, she noticed the swelling 2 months before. It was insidious in onset and slowly progressive to the present size. There were no contributing medical and dental histories. Upon general examination the patient was moderately built and nourished. Her vitals were normal. Extraoral examination revealed facial asymmetry due to the swelling present over the left malar region [figure 1].

a) and b) extraoral images showing facial asymmetry due to the diffuse swelling in left malar region obliterating the nasolabial fold 
Intraorally diffuse swelling was noted from $22-$ 27 region causing obliteration of the buccal mucosa. The overlying mucosa was intact. On palpation it was soft in consistency and compressible with mild tenderness present. Examination of the teeth showed missing 13 and retained 53 (Figure 2). Consent was obtained from the patient's father and wide bore needle aspiration was done which yielded blood tinged cystic fluid [Figure 2].

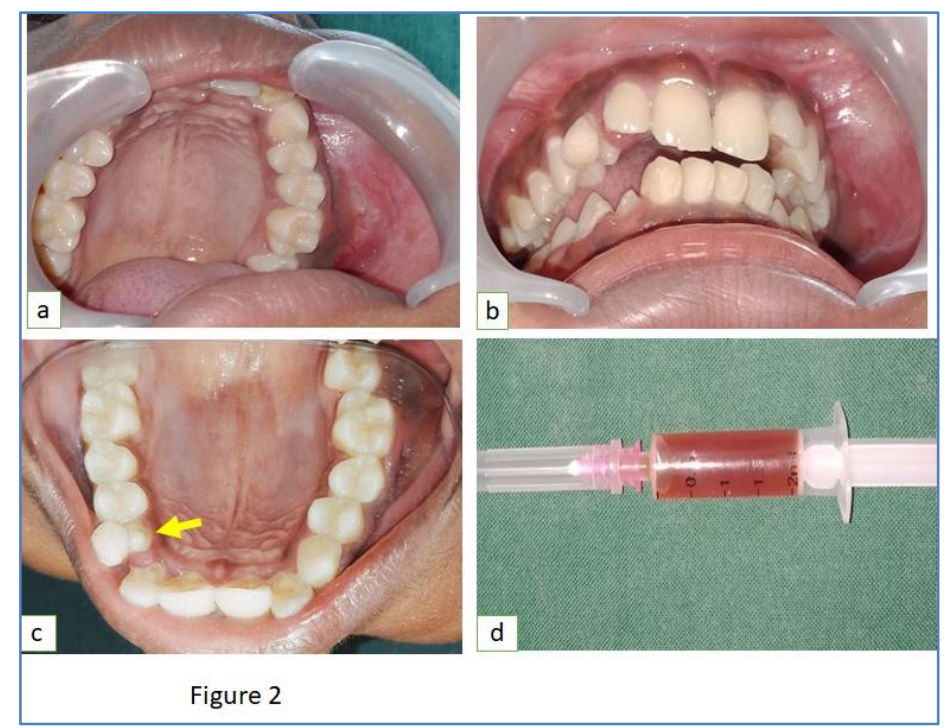

a) and b) intraoral view showing swelling in relation to $24,25,26,27$ causing obliteration of the buccal vestibule,

c) Palatal view showing retained deciduous 53 and crown portion of 13,

d) Wide bore needle aspiration of $2 \mathrm{ml}$ blood tinged cystic fluid

So, considering her age and based upon the history and clinical examination a provisional diagnosis of Odontogenic cysts was given. Further the patient was subjected for radiological investigations. Radiographically, Intra Oral Periapical Radiograph (IOPA) revealed widening of PDL space in, 25, 26 and root resorption of 27. Cross sectional occlusal radiograph of Maxilla revealed impacted mesiodens and 13 with a retained deciduous 53 and a radiolucent lesion with sclerotic border noted near 27. Panoromic view showed haziness over left maxillary region while comparing to the right side and displacement of tooth bud 28 . Other findings included developing tooth buds 18,38,48, impacted mesiodens between 11 and 21 , teeth with open apex in 44,45,47,34,35,37,27,17, retained deciduous 53 and impacted 13 [figure 3].

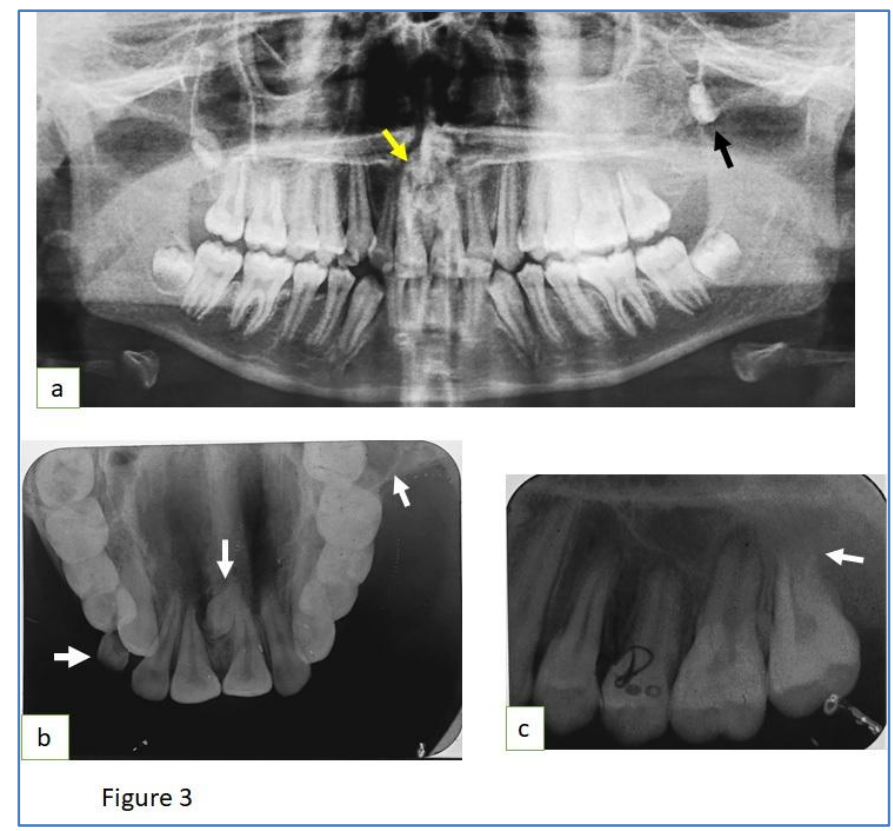

a) Panoromic view showing haziness over left maxillary sinus, black arrow indicating the lesion has displaced the tooth bud 28, yellow arrow indicates the impacted mesiodens. Teeth with open apex 33,34,35,37,43,44,45,47,27,17. Tooth buds $18,28,38,48$. 
b) Cross sectional maxillary occlusal radiograph shows impacted mesiodens, white arrow near 27 shows the radiolucent lesion surrounded by radiopaque border and white arrow on the right side of the radiograph shows impacted 13 and retained deciduous 53.

c) IOPA ,25, 26 showing widening of PDL space and root resorption of 27

CT coronal section reveals soft tissue density lesion in left maxilla causing displacement of tooth bud 28 and destruction of the posterior wall of left maxillary sinus. Thick corticated rim was seen within the soft tissue lesion of the left maxillary sinus. CT axial section shows destruction of buccal cortical plate in relation to 24, 25, 26 and 27 and the adjacent soft tissue invasion of the lesion. Invagination of the sinus cavity by the lesion and the presence of corticated border of the lesion within the sinus cavity [figure 4].

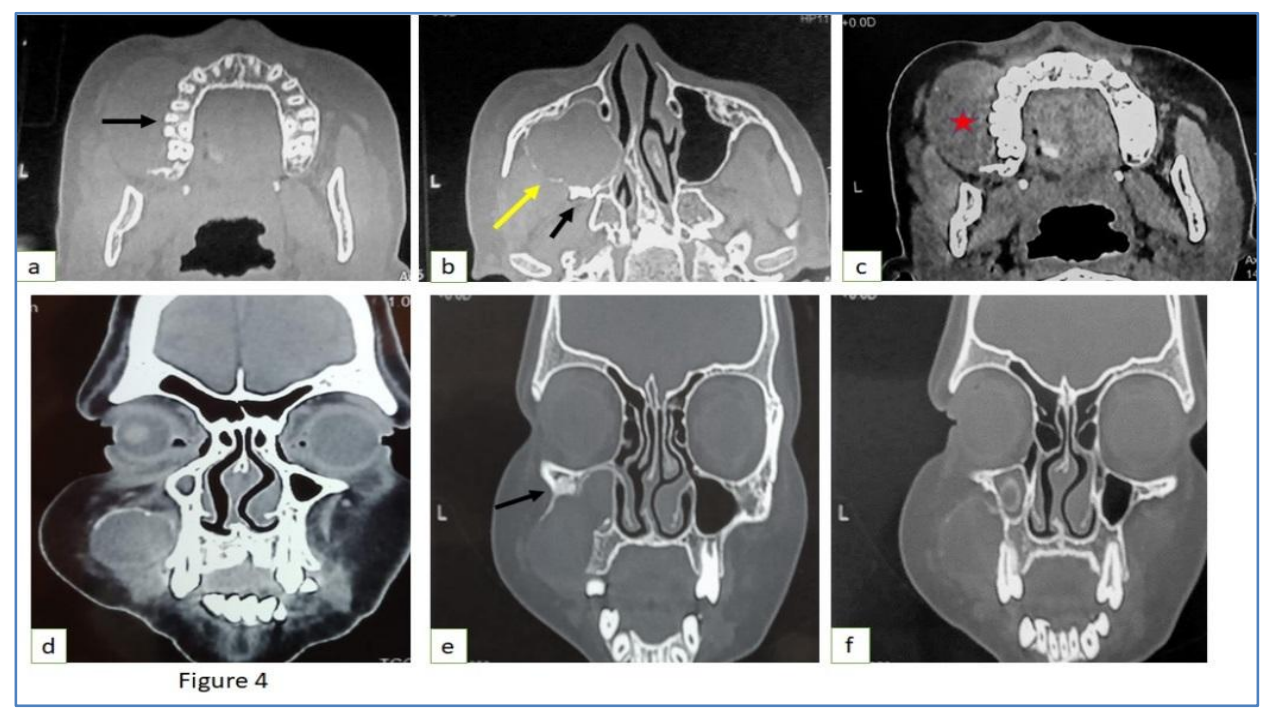

a. CT axial view at the level of maxillary alveolar ridge indicates the destruction of the buccal cortical plate in relation to $25,26,27$ and adjacent soft tissue invasion of the lesion

b. CT axial view at the level of the maxillary sinus reveals complete obliteration of the maxillary sinus by the lesion. The black arrow indicates the displacement of the tooth bud 28 due to the expanding nature of the lesion and the yellow arrow denotes the corticated border of the lesion within the sinus cavity

c. The asterisk symbol in CT soft tissue window axial section indicating the lesion

d. soft tissue window CT coronal section at the level of maxillary canine region showing a well- defined soft tissue density lesion in left maxilla with discontinuity in its corticated border

e. Bone window CT coronal section at the level of maxillary first premolar region shows the invagination of the cystic lesion into the sinus cavity and, also destruction of the posterolateral wall of left maxillary sinus and displacement of tooth bud 28 (indicated by black arrow)

f. Thick corticated rim present within the soft tissue density lesion

So, by analysing the radiographic features COC was considered, differential diagnosis included Dentigerous cyst, Calcifying Epithelial Odotogenic tumor, and Adenomatoid Odontogenic tumor were included.for arriving at the final diagnosis patient was planned for Histopathological examination (HPE). Incisional biopsy was done, and the histology was suggestive of a cystic lesion with an epithelial lining that is characterized by the presence of cuboidal to columnar basal cells and loosely arranged superficial stellate reticulum like cells. Anucleate eosinophilic ghost cells forming large amorphous sheets are seen within the epithelial lining. Calcifications are seen within the epithelium and the inflamed fibrous cyst wall and thus confirming Calcifying Odontogenic Cyst [figure 5].

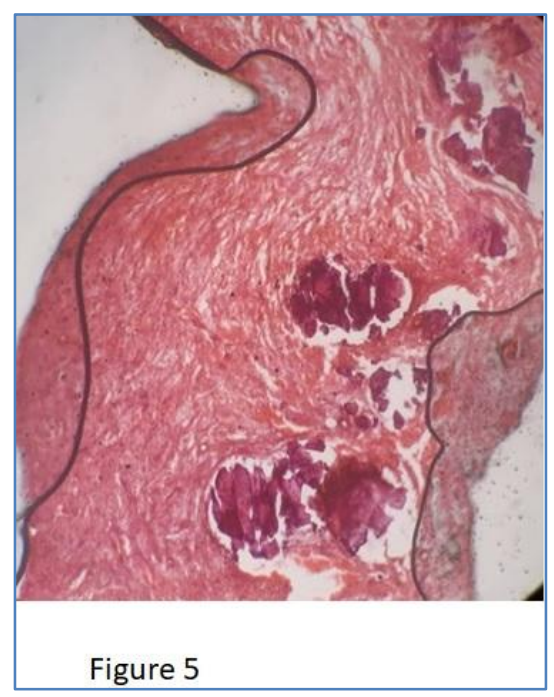


Photomicrograph picture of $\mathrm{H} \& \mathrm{E}$ stained histological section in $10 \mathrm{x}$ view showing an epithelial lining characterized by cuboidal to columnar basal cells and loosely arranged superficial stellate reticulum like cells. Anucleate eosinophilic ghost cells forming large amorphous sheets are seen within the epithelial lining. Calcifications are seen within the epithelium and the inflamed fibrous cyst wall

Once the final diagnosis was confirmed patient was referred for surgical management.

\section{DISCUSSION}

COC is a unique lesion that shows the characteristics of both a solid neoplasm and a cyst, therefore it was characterized as a distinct entity by Gorlin. Most of these lesions are non-neoplastic. Other terminologies used are dentinogenic ghost cell tumor, calcifying cystic odontogenic tumor, atypical adamantinoma [6]. COC displays a variety of clinical, pathological and histological characteristics. It is clinically and radiologically similar to other lesions such as Calcifying Epithelial Odontogenic tumor, Ameloblastic Fibro Odontoma, Adenomatoid Odontogenic Tumor, Dentigerous cyst and other Odontogenic cysts [7]. Praetorius et al. at first classified these lesions into cystic type and neoplastic type. The subdivisions of Cystic type include simple cystic type, odontoma producing type and ameloblastomous proliferating type, whereas the neoplastic type compromises solid lesion such as Dentinogenic Ghost cell tumor [8]. Radiographically in early stages the lesion will appear completely radiolucent, as it matures calcifications form that yields a mixed radiopaque and radiolucent lesion [9]. The presence of calcifications is a very unique finding in COC. The frequency of enveloped teeth in COC is roughly $32 \%$. According to Lida et al it was concluded that most of the COCs enveloping the tooth crown were noticed in the posterior part of the jaws [ 10 ].This was consistent with the present case where the tooth bud 28 was displaced due to the lesion. Also, in the present case there was impacted mesiodens and permanent right maxillary canine. Evidence of root resorption was noted in 27. According to Praetorius et al the intraosseous type of cystic lesion erodes the bone rather than causing expansion and $75-77 \%$ of the lesions causes root resorption due to the fact these lesions involve the apices of the teeth [11]. Treatment usually includes surgical enucleation and curettage [12]. The prognosis for a patient with COC is good, only a few recurrences have been reported. When a COC is associated with other odontogenic tumors like ameloblatoma the treatment and prognosis are same as the associated tumors [13]

\section{CONCLUSION}

Calcifying Odontogenic cyst is a rare lesion which shows clinico radiological diagnosis similar to that of other odontogenic cysts, so the diagnosis has to be confirmed by histopathological examination. The recurrence reported is about 5\% [14]. Though the prognosis is good for COC follow up of the patient is essential to prevent recurrent cases from malignant transformation

\section{ACKNOWLEDGEMENTS}

We sincerely acknowledge to the Department of Oral and Maxillofacial Pathology for their histopathological support

\section{REFFERENCE}

1. Rojo, R., Prados-Frutos, J. C., Lázaro, I. G., \& Alonso, J. H. (2017). Calcifying odontogenic cysts. Journal of stomatology, oral and maxillofacial surgery, 118(2), 122-124.

2. Kim, Y., Choi, B. E., \& Ko, S. O. (2016). Conservative approach to recurrent calcifying cystic odontogenic tumor occupying the maxillary sinus: a case report. Journal of the Korean Association of Oral and Maxillofacial Surgeons, 42(5), 315-320.

3. Arruda, J. A., Silva, L. V., Silva, L., Monteiro, J. L., Álvares, P., Silveira, M., \& Sobral, A. P. (2018). Calcifying odontogenic cyst: A 26-year retrospective clinicopathological analysis and immunohistochemical study. Journal of clinical and experimental dentistry, 10(6), e542.

4. Santos, H. B. D. P., de Morais, E. F., Moreira, D. G. L., Neto, L. F. D. A., Gomes, P. P., \& Freitas, R. D. A. (2018). Calcifying odontogenic cyst with extensive areas of dentinoid: Uncommon case report and update of main findings. Case reports in pathology, 2018.

5. Mulvihill, C., Ní Mhaolcatha, S., Brady, P., McKenna, J., Sleeman, D., \& Fitzgibbon, J. (2020). Calcifying odontogenic cyst: A case report. Oral Surgery, 13(2), 177-181.

6. Figueiredo, N. R., Meena, M., Dinkar, A. D., \& Khorate, M. (2014). Calcifying epithelial odontogenic cyst of the mandible. Journal of oral research and review, 6(2), 57.

7. Kamal, F., Deepthi, M., \& Raghunath, V. (2019). Calcifying odontogenic cyst: Report of a case with unique features. SRM Journal of Research in Dental Sciences, 10(2), 110.

8. Sonone, A., Sabane, V. S., \& Desai, R. (2011). Calcifying ghost cell odontogenic cyst: report of a case and review of literature. Case reports in dentistry, 2011.

9. Gamoh, S., Akiyama, H., Furukawa, C., Matsushima, Y., Iseki, T., Wato, M., \& Shimizutani, K. (2017). Calcifying cystic odontogenic tumor accompanied by a dentigerous cyst: A case report. Oncology letters, 14(5), 57855790 .

10. Iida, S., Fukuda, Y., Ueda, T., Aikawa, T., Arizpe, J. E., \& Okura, M. (2006). Calcifying odontogenic 
cyst: radiologic findings in 11 cases. Oral Surgery, Oral Medicine, Oral Pathology, Oral Radiology, and Endodontology, 101(3), 356-362.

11. Daniels, J. S. M. (2004). Recurrent calcifying odontogenic cyst involving the maxillary sinus. Oral Surgery, Oral Medicine, Oral Pathology, Oral Radiology, and Endodontology, 98(6), 660-664.

12. de Moraes, A. T. L., Soares, H. A., Pinheiro, J. D. J. V., \& Ribeiro, A. L. R. (2020). Marsupialization before enucleation as a treatment strategy for a large calcifying odontogenic cyst: Case report. International Journal of Surgery Case Reports, 67, 239-244.

13. Day, T. A., \& Girod, D. A. (2005). Oral cavity reconstruction. CRC Press.

14. Sumathy, M. P., Kuzhali, S., Harishnath, V., Gnanadeepam, S., Selvajothi, P., \& Ramyadevi, S. Cystic lesion in the maxilla: A case report of calcifying odontogenic cyst with review of literature. 